\title{
Experimental characterization of a space charge induced modulation in high-brightness electron beam
}

\author{
T. Shaftan \\ National Synchrotron Light Source, Brookhaven National Laboratory, Upton, New York, 11973, USA \\ Z. Huang \\ Stanford Linear Accelerator Center, 2575 Sand Hill Road, Menlo Park, California, 94025, USA
}

(Received 19 November 2003; published 26 August 2004)

\begin{abstract}
We present the experimental investigation of a collective effect driving strong modulation in the longitudinal phase space of a high-brightness electron beam. The measured beam energy spectrum was analyzed in order to reveal the main parameters of modulation. The experimental results were compared with a model of space-charge oscillations in the beam longitudinal phase space. The measurements and analysis allowed us to determine the range of the parameters of the observed effect on the modulation dynamics and illustrate its potential impact on short-wavelength free-electron laser performance.
\end{abstract}

DOI: 10.1103/PhysRevSTAB.7.080702

PACS numbers: 41.75.Fr, 41.60.Cr, 52.59.Sa

\section{INTRODUCTION}

During the past decade a growing demand for a new generation of light sources has initiated research and development on high-gain free-electron lasers (FEL) [1]. New light sources are required, having unprecedented brightness along with flexible temporal properties of radiation in the extreme ultraviolet to $\mathrm{x}$-ray wavelength range [2-5]. These requirements place serious constraints on the allowable quality of the "active medium" in accelerators-FEL drivers. For successful operation of high-gain FEL, electron beam must have a very small normalized emittance $(\sim 1 \mu \mathrm{m})$ and a very high peak current (approximately a few kA).

Problems in the generation and transport of highbrightness beams led to intensive investigation of possible instabilities, capable of degrading the beam quality. The studies revealed several new effects, specific for highbrightness machines. One of these, coherent synchrotron radiation (CSR) in the bunch compressors, appeared to be able to amplify any microbunching in the beam longitudinal phase space [6-9]. In addition, longitudinal space charge and linac wakefield can also drive the microbunching instability [10-12]. These effects may be very large for short-wavelength FELs when multistage bunch compression is incorporated. Possible solutions for suppressing this instability have been proposed and studied $[2,12,13]$.

Several years ago two FEL facilities reported observations of a new complicated phenomenon. At the commissioning stage of Deep Ultraviolet FEL (DUV-FEL at NSLS, BNL) and TESLA Test Facility (TTF) FEL (DESY), strong modulation of the electron bunch energy spectra was observed [14,15]. During measurements of the bunch length using the so-called zero-phasing method [16] the energy spectra of the compressed bunch exhibited spiky structure with subpicosecond spike separation. To reveal the main properties of the structure, several experiments were proposed and performed [17-19]. In this paper we discuss the results of an experiment at the DUV-FEL facility and compare them with a simple model we developed.

The model [20] is based on the longitudinal spacecharge effect, which drives small nonuniformities in the longitudinal bunch density into energy modulation along the bunch. Small density clusters in the electron bunch create longitudinal space-charge field that accelerates particles at the head of the cluster and decelerates particles at the tail. This initiates space-charge oscillations in the bunch as it travels along the accelerator. As a result, the longitudinal phase-space distribution of the electron bunch at the end of the accelerator is strongly distorted. This effect is emphasized in the high-peak current regime required for achieving high gain in a singlepass FEL.

Analysis of the model predicts sensitivity of the effect to the beam parameters. In the experiment described in this paper, we studied the dynamics of the modulation with respect to the beam energy, maintaining all other beam parameters constant.

In Sec. II we describe the experimental setup and present the results of the experiment. In Sec. III we discuss the numerical simulation developed according to the model. Using the simulation we calculated the amplitudes of energy modulation for different beam parameters and compared them with measured data. In Sec. IV we summarize the results of this work and discuss their significance on future $\mathrm{x}$-ray FELs.

\section{EXPERIMENTAL DESCRIPTION AND DATA ANALYSIS}

The experiment was performed at the DUV-FEL linear accelerator [21] (Fig. 1). An electron bunch with about 


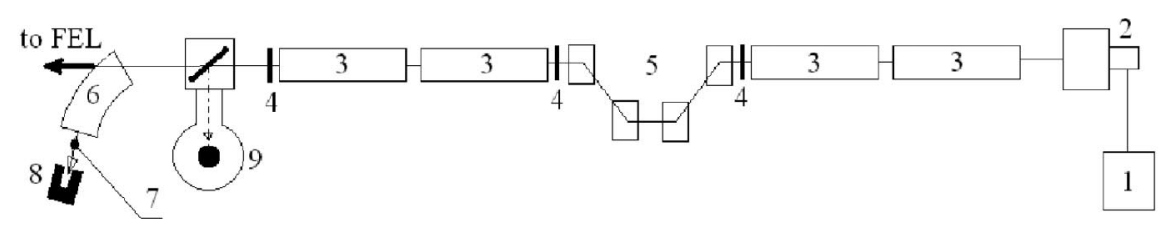

FIG. 1. The DUV-FEL accelerator layout. 1: rf gun drive laser; 2: rf gun with focusing solenoid; $3: 3 \mathrm{GHz}$ linac tank; 4 : focusing triplets; 5: magnetic chicane; 6: spectrometer dipole; 7: beam monitor; 8: beam dump; 9: coherent transition radiation station. The beam energy is $6 \mathrm{MeV}$ after the rf gun and $72 \mathrm{MeV}$ after the second linac tank.

$200 \mathrm{pC}$ of charge was generated in a rf gun, driven by a 1.3-mm-long laser pulse. The bunch was accelerated up to an energy of $71.5 \mathrm{MeV}$ and compressed in a magnetic chicane from a length of $1.2 \mathrm{~mm}$ FWHM down to $280 \mu \mathrm{m}$, which corresponds to 4 times the increase in the bunch peak current. The experimental setup consisted of two $3 \mathrm{GHz}$ traveling wave accelerating sections following the chicane and an energy spectrometer containing a bending dipole with a beam profile monitor (Fig. 1).

The combination of amplitude and phase of the first section rf wave was chosen in a way not only to change the energy of the beam centroid, but also to simultaneously minimize the correlated energy spread remaining after compression. The second accelerator section was carefully calibrated to provide a known amount of energy chirp, which the spectrometer transformed into a spatial distribution on the downstream monitor, viewed with a video camera.

Examples of chirped-beam energy (CBE) spectra are shown in Fig. 2. The local brightness in the image is linearly proportional to the local amount of charge. The horizontal coordinate of the beam image scales with energy; the position of the image centroid is defined by the average beam energy. Electrons, located in the head (tail) of the bunch, gain (lose) energy while traveling through the second accelerator section, but the energy of the beam centroid stays constant. Therefore the bunch gets dispersed along the horizontal axis of the monitor and the head (tail) of the bunch gets mapped on the right (left) side of the monitor screen. This setup is a particular implementation of the so-called "zero-phasing" method of bunch length measurement [16]. Since we can measure both the energy and the correlated energy chirp along the bunch, this method allows us to calibrate the energy axis in units of time in the bunch rest frame.

The structure seen in the CBE spectrum in Fig. 2 was the subject of the investigation. One could argue that the structure in the higher energy image looks sharper and therefore that the modulation amplitude significantly increases with energy. Fourier analysis of the energy spectrum confirms this conclusion: the spectral intensity at modulation frequencies is indeed larger for higher energy. Surprisingly, this conclusion is wrong. Careful analysis of the images in Fig. 2 shows that the product of modulation amplitude and modulation wave number for $50 \mathrm{MeV}$ is bigger than the value of energy chirp imparted by the
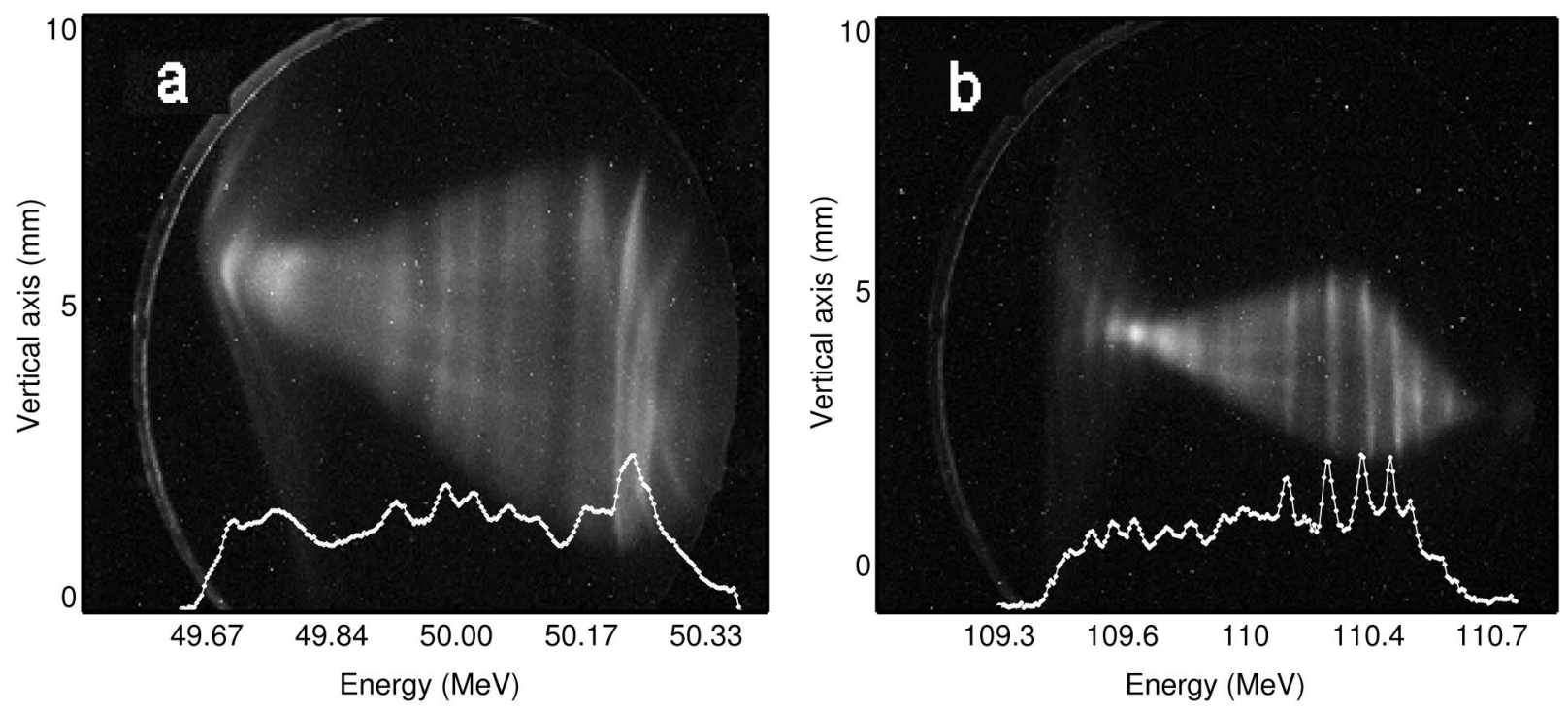

FIG. 2. Monitor after the spectrometer dipole: transverse beam distributions (images) and chirped bunch energy (CBE) spectra (curves) for (a) 50 and (b) $110 \mathrm{MeV}$. The CBE spectrum is a projection of the image onto the horizontal axis. 

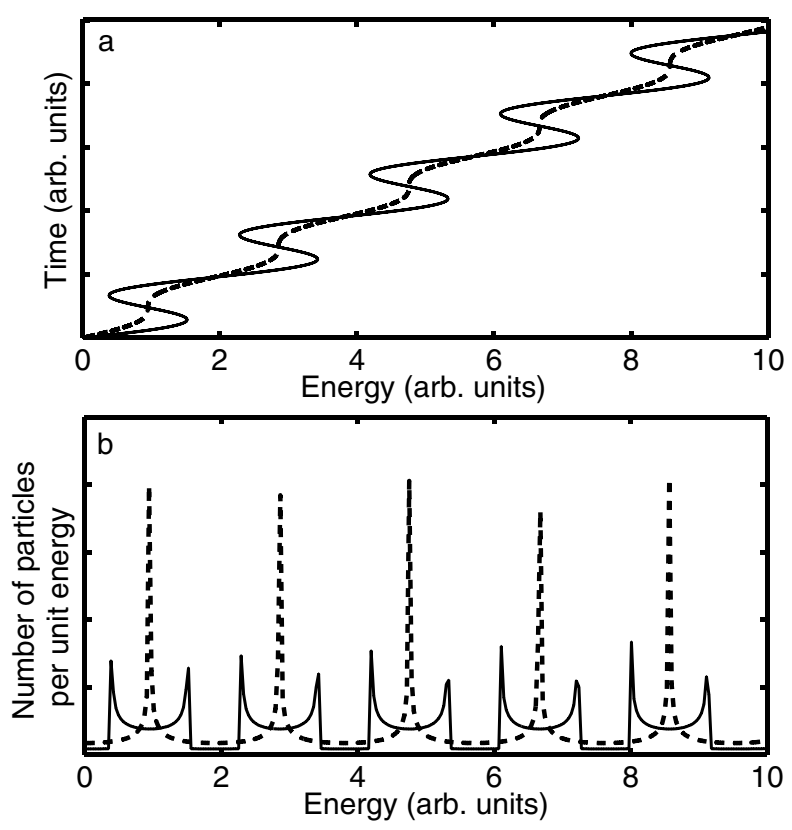

FIG. 3. Sketch of the beam longitudinal phase space (a) and its projections on the energy axis, or CBE spectrum (b). Intrinsic energy spread is neglected. Two beams are shown, one with a larger (solid) and a second with a smaller (dashed) energy modulation amplitude. The beam with a larger amplitude represents the overmodulated case, i.e., energy along the bunch is not a monotonic function of time. The energy spectrum of the beam with a smaller modulation amplitude looks significantly more "spiky."

second accelerator section. This causes "overmodulation" of the structure, which is sketched in Fig. 3. Therefore the CBE spectrum looks smoother for lower energy. In addition, as a component of the spectrometer resolution, relative intrinsic energy spread increases at lower energy, which furthermore reduces the contrast of the image.

As we have shown in Ref. [20], the observed structure must contain a contribution of modulation in longitudinal density, besides energy modulation. Direct experimental determination of the content of longitudinal modulation is difficult because of lack of high-resolution diagnostics in the femtosecond time range. To determine whether the observed structure is dominated by energy modulation, we performed two additional experiments [18,19].

Using three focusing solutions with different beam envelopes $(0.25,0.5,1.0 \mathrm{~mm} \mathrm{rms})$ we measured the sensitivity of the modulation to the change in beam size along the zero-phasing section of the accelerator. It was explicitly shown that the strong modulation, present when the beam size is small, almost vanishes at a larger beam cross section. ${ }^{1}$ The observed structure, if caused by modu-

\footnotetext{
${ }^{1}$ Note that the change of beam size along accelerator did not affect spectrometer resolution. Also beam size variation occurred only after compressor.
}

lation in the longitudinal density should not be very sensitive to the change in transverse beam dimensions. On the contrary, space-charge-induced energy modulation can have a strong dependence on the beam size. In the next experiment we measured coherent transition radiation (CTR) power from electron bunches with a drastically different modulation amplitude under the same experimental conditions. Measured values of power for various CTR wavelength ranges did not differ for the two cases, indicating that the spectral content of density modulation is comparable in both cases. These experiments have confirmed the conclusion that the observed structure is dominated by energy modulation instead of current modulation.

Manipulating with the first accelerator section, we varied the beam energy from 50 to $110 \mathrm{MeV}$. All other beam parameters, including bunch length, average rms transverse sizes (measured as $\sigma_{x} \approx \sigma_{y} \approx 450 \mu \mathrm{m}$ ), charge, etc., were maintained nearly constant during the measurements.

A large number of CBE spectra were recorded for every value of energy. Experimental data were analyzed in order to retrieve two main parameters of the structure: time period and amplitude. A detailed description of the modulation analysis is given in [22]. For this particular experiment we picked two modulation periods from each of the recorded CBE spectra and averaged over each set of measurements related to the same energy value.

The number of modulation periods and the modulation wavelength versus energy are plotted in Fig. 4. The frequency bandwidth of modulation lies in the terahertz range. Note that the average modulation wavelength decreases with energy while the number of modulation

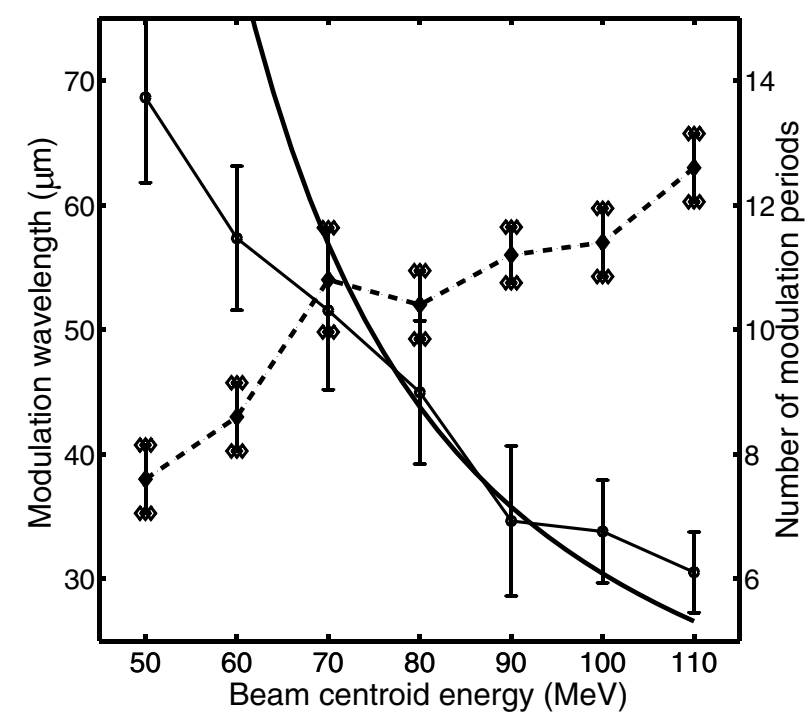

FIG. 4. Measured dependencies of the average modulation wavelength (solid) and the average number of modulation periods (dashed) versus beam centroid energy. The solid curve represents the result of analytic calculation (see text for details). 


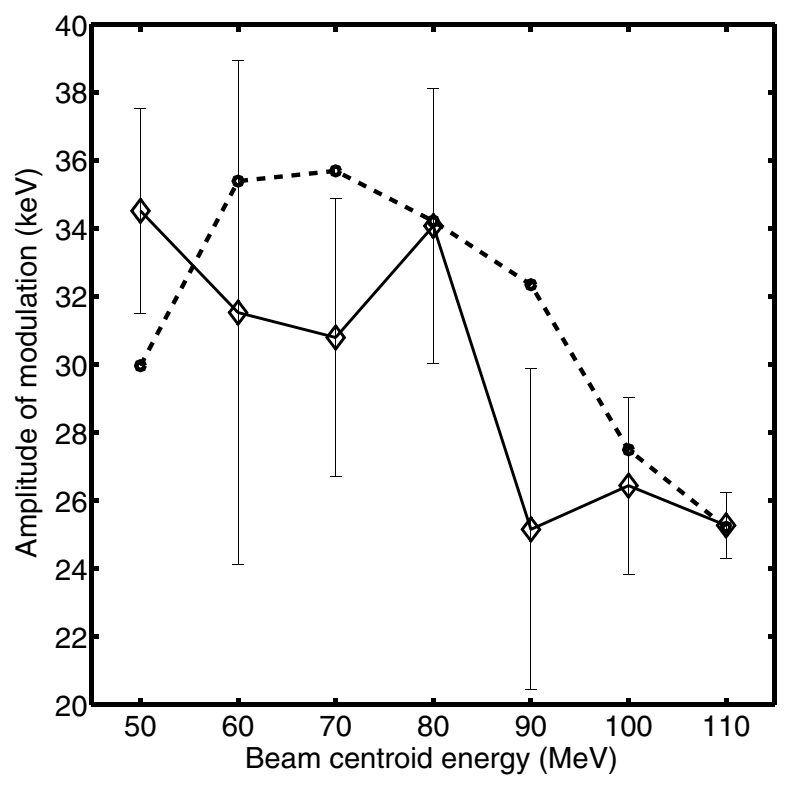

FIG. 5. Measured dependence of the energy modulation amplitude on beam centroid energy. The dashed curve is the simulation result of space-charge induced energy modulation with about $\pm 3 \%$ of initial density modulation at the measured modulation wavelength.

periods increases with energy, so that their product, bunch length, is kept constant in this experiment.

The dependence of the modulation amplitude on energy is shown in Fig. 5. The average amplitude of modulation is found to be around $30 \mathrm{keV}$ or $4 \times 10^{-4}$ at an average energy of $80 \mathrm{MeV}$. Although we do not have direct measurements of the intrinsic energy spread, both the recent TTF photoinjector measurement [23] and the DUV-FEL high-gain harmonic generation experiment [24] indicate that it is extremely small after typical photoinjectors. PARMELA space-charge simulation shows an rms value of $\sim 2 \mathrm{keV}$ at about $200 \mathrm{pC}$ [25]. This intrinsic energy spread is increased to about $8 \mathrm{keV}$ after the chicane (with a compression factor of $\sim 4$ ) due to the conservation of the longitudinal phase space. Therefore, modulation structure by far dominates uniform beam distribution, and the spikes in the images corresponding to $\mathrm{CBE}$ spectra look very sharp. Since the beam has a larger energy modulation than its intrinsic energy spread, it may be subject to the microbunching instability if under additional compression. For the DUV-FEL, this beam is not further compressed, and the measured energy modulation amplitude is well within the FEL tolerance determined by the FEL Pierce parameter [26] (about $2.5 \times 10^{-3}$ or $440 \mathrm{keV}$ at $175 \mathrm{MeV}$ ).

\section{COMPARISON WITH A MODEL}

To reveal the dependence of the modulation on the beam energy and to explain the observations described above, we constructed a model based on the longitudinal space-charge (LSC) effect [20]. LSC is considered to be the dominant collective effect in this experiment because the longitudinal impedance of the accelerator structure generally diminishes at very high frequencies (higher than $\gamma c / a$, where $a$ is the vacuum chamber radius) $[11,12]$. CSR impedance can be stronger than LSC at the DUV-FEL bunch compressor chicane and even at the spectrometer dipole. However, the density modulation of the electron beam is strongly Landau damped by the emittance effect in bends where the CSR impedance may be important [7-9]. The measured normalized emittance is $\gamma \varepsilon=3 \mathrm{~mm} m$ rad at the DUV-FEL. The chicane consists of four dipoles of length $L_{d}=0.19 \mathrm{~m}$ and bending radius $R=1.1 \mathrm{~m}$. Assuming that the initial beta function is $\beta_{0}=5 \mathrm{~m}$, the density modulation in the chicane (at the energy $\gamma m c^{2}=72 \mathrm{MeV}$ ) is suppressed for an initial wavelength smaller than $2 \pi\left(\varepsilon \beta_{0}\right)^{1 / 2} L_{d} / R \approx 360 \mu \mathrm{m}$. Taking into account a factor of 4 compression after the chicane, we conclude that the observed modulation structures of the compressed beam for a modulation wavelength $\lambda<90 \mu \mathrm{m}$ cannot be driven significantly by CSR, in agreement with the small CSR microbunching gain across the chicane as computed in Ref. [17]. Similarly, no significant energy and density modulations can be induced by CSR in the spectrometer dipole at these short wavelengths. Note that the rms electron beam size varies from about $300 \mu \mathrm{m}$ to $1 \mathrm{~mm}$ in the chicane and is still within the transverse coherence length of the radiation $\left[\left(\lambda^{2} R\right)^{1 / 3} \approx 2 \mathrm{~mm}\right.$ for $\left.\lambda \sim 100 \mu \mathrm{m}\right]$. Thus, the onedimensional CSR impedance used in Refs. [7-9,17] is a valid approximation. Finally, we observe that the sensitive dependence of the modulation amplitude on the average beam size in the rf zero-phasing section $[18,19]$ is consistent with the LSC model.

In the frame of the model the first accelerating section was represented as a distributed acceleration together with a space-charge force, acting at discrete locations along the beam path. The following drift was treated in the same way, except that the bunch energy was kept constant. The drift distance was chosen according to the real length between the first accelerator section and the beam monitor. An ensemble of microparticles was tracked through the model setup for every experimental value of the bunch energy. The free-space, longitudinal space-charge impedance per unit length is approximately given by [20,27]

$$
Z(\lambda)=i \frac{Z_{0} \lambda}{2 \pi^{2} r_{b}^{2}}\left[1-\frac{2 \pi r_{b}}{\gamma \lambda} K_{1}\left(\frac{2 \pi r_{b}}{\gamma \lambda}\right)\right]
$$

where $Z_{0}=377 \Omega$ is the free-space impedance, $r_{b}$ is the beam radius for a uniform cross section and is taken to be roughly $\left(\sigma_{x}+\sigma_{y}\right)$ for a Gaussian or a parabolic cross section, and $K_{1}$ is the modified Bessel function. Performing the Fourier transform of (1) we obtain longitudinal space-charge field per unit length for the current, containing modulation at a single frequency. The 
action of the space-charge force was represented as a change of energy in a local slice along the bunch, due to interaction from the neighboring slices. The tracking procedure allowed us to obtain the longitudinal phasespace distribution of the particles at consecutive locations along the beam path. The final phase-space distributions were observed at the actual distance from the end of the compressor down to the monitor location.

Note that only the part of the accelerator starting at the end of the compressor was taken into consideration in the model. This was done because observed CBE spectra for the bunch with the compressor turned off exhibited almost no spiky structure. Thus the modulation under study was assumed to be specific only for the regime with a high-peak current, or the compressed bunch regime.

Our experimental setup did not have the ability to characterize the longitudinal phase space right at the end of the compressor. The following assumption was chosen for the initial conditions for particle distribution. We assumed that the initial phase-space distribution included a certain amount of density modulation, or microbunching. At the same time any initial energy modulation, which might be present in the beam before the compressor, was neglected.

A simulation based on the described model demonstrated the development of energy modulation, seeded by initial density modulation. The final modulation amplitude depends on the modulation wavelength and scales linearly with the amplitude of initial density modulation.

We used the simulation for comparison of the predicted energy modulation amplitude (Fig. 5) with quantities derived from experimental data [22]. As the experiment revealed a dependence of the measured modulation wavelength on the beam energy, we introduced initial density modulation at the average modulation wavelength specific for every experimental value of energy. Simulation demonstrated that reconstructed amplitude of energy modulation (Fig. 5) corresponds to about $3 \%$ of initial density modulation amplitude, comparable with the measured fluctuations of $\mathrm{rf}$ gun drive laser intensity along the $266 \mathrm{~nm}$ laser pulse [18]. We may conclude that nonuniformities in the longitudinal bunch density in the range of only a few percent can cause strong modulation of energy along the bunch.

The above analysis suggests that the initial density spectrum is quite uniform over a wide range of wavelengths (from 30 to $70 \mu \mathrm{m}$ ). Nevertheless, the final energy spectrum has a preferred average modulation wavelength at a given electron energy, as shown in the measured data of Fig. 4. This can be explained by the space-charge dynamics in the zero-phasing section. The ratio of the amplitudes of final energy modulation to the initial density modulation is determined by [20]

$$
\left[\frac{\gamma I_{0}}{I_{A}} \frac{2 \lambda Z(\lambda)}{Z_{0}}\right]^{1 / 2} \sin \left(\frac{\Omega_{\mathrm{SC}} L}{c}\right),
$$

where $I_{0} \approx 200 \mathrm{~A}$ is the peak current, $I_{A} \approx 17 \mathrm{kA}$ is the Alfvén current, $L$ is the distance between compressor and beam monitor, and the space-charge oscillation frequency is

$$
\Omega_{\mathrm{SC}}=c \sqrt{\frac{8 \pi^{2} I_{0}}{\gamma^{3} I_{A}} \frac{|Z(\lambda)|}{\lambda Z_{0}} .}
$$

At lower energies, the energy modulation is maximized near the modulation wavelength that has the space-charge oscillation phase $\Omega_{\mathrm{SC}} L / c \approx \pi / 2$. At higher energies, the beam is more rigid, and the space-charge oscillation phase advance is small. The maximum energy modulation is then determined by the maximum of the space-charge impedance given in Eq. (1). At the maxima of Eq. (2), we plot the modulation wavelength as a function of the beam energy in Fig. 4 and note the reasonable agreement with the measured average modulation wavelength.

Strictly speaking, Eqs. (2) and (3) could be used only for a constant energy beam. In reality, the beam energy changes as the beam travels along the first accelerating section, although the section (of length about $3 \mathrm{~m}$ ) is significantly shorter than the drift (of length about $12 \mathrm{~m}$ ) where the energy is constant. Numerical simulation discussed at the beginning of this section was used to study the beam energy change and its induced modulation wavelength dependence. We simulated a propagation of the beam with the initial density modulation seeded by a single frequency in the range from 10 to $100 \mu \mathrm{m}$. Figure 6 shows the spectral shapes of final density and energy modulations at two extreme energies of the experiment, assuming the initial density modulation amplitude of about $3 \%$ at all wavelengths. The maximum energy

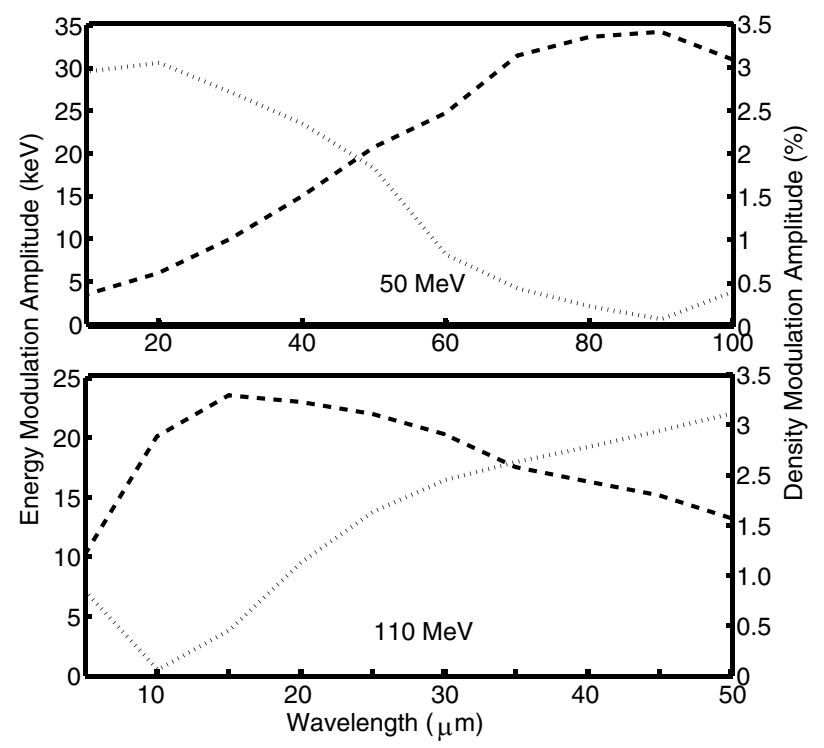

FIG. 6. Numerical simulation results. The spectral shapes of final density (dotted curves) and energy (dashed curves) modulation for 50 and $110 \mathrm{MeV}$ assuming $\pm 3 \%$ initial density modulation at all wavelengths. 
modulation occurs at a much longer modulation wavelength at $50 \mathrm{MeV}$ than at $110 \mathrm{MeV}$, in general agreement with the measurements and the analytical prediction. The difference in modulation wavelengths between measurements and theory/simulation at the low-energy side (Fig. 4) may be due to the complicated (and unknown) longitudinal phase-space distribution.

Thus, space-charge oscillations at different frequencies accumulate different phase advances while the bunch travels down to an accelerator. The space-charge impedance represents a broadband filter that determines the relative strength of energy and density modulation amplitudes as a function of frequency (Fig. 6). Since spectral function of such a "filter" is energy dependent, the beam energy spectrum at the end of accelerator exhibits frequency content that varies with energy. In order to further illustrate this point we have simulated propagation of the beam with the initial current spectrum containing $\pm 3 \%$ density modulation at six separate wavelengths $(20,30$, $40,50,60$, and $70 \mu \mathrm{m}$ ). The results of the simulation for the energies of 50 and $110 \mathrm{MeV}$ are shown in Fig. 7. One can observe approximately two times fewer spikes in the energy spectrum for the $50 \mathrm{MeV}$ beam versus the $110 \mathrm{MeV}$ case. Again, this agrees with Fig. 6, where the high frequency part of energy modulation spectrum is attenuated for the $50 \mathrm{MeV}$ beam. In order to better reproduce the measured energy spectra (Fig. 2) we have to take into account the photocathode drive laser spectrum and follow

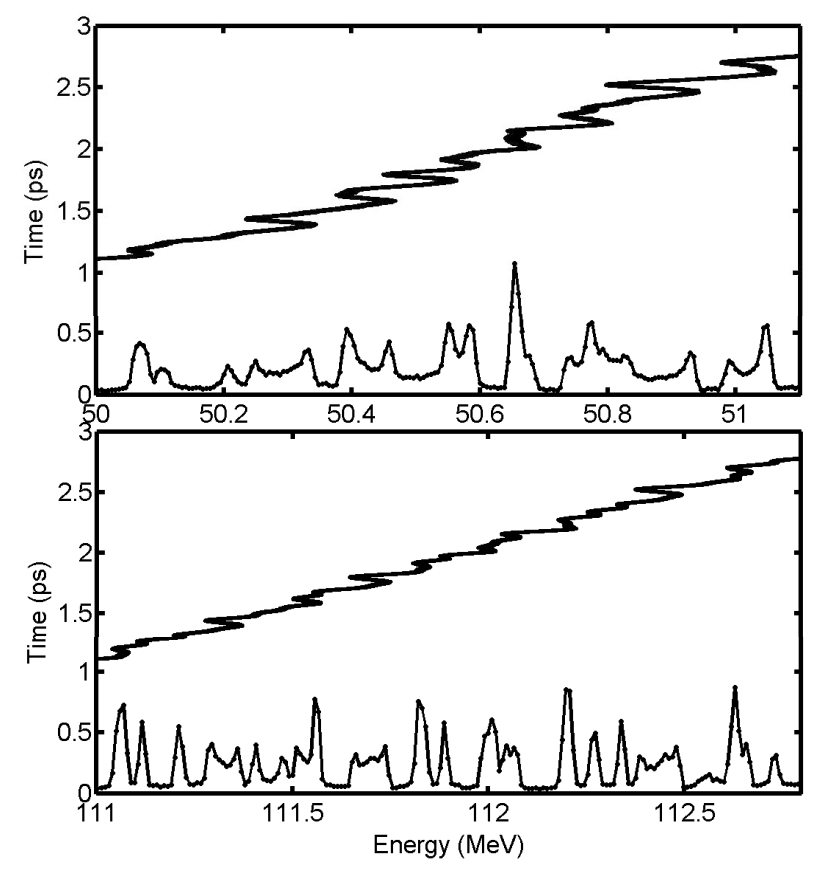

FIG. 7. Numerical simulation results. The final phase-space and energy spectra for 50 and $110 \mathrm{MeV}$ beams assuming that the initial density modulation spectrum consists of six individual wavelengths $(20,30,40,50,60$, and $70 \mu \mathrm{m})$ with the same amount of density modulation $( \pm 3 \%)$. the beam modulation spectrum throughout the entire DUV-FEL beam lines, as shown in Fig. 1.

\section{CONCLUSION}

This paper describes the experimental characterization of a space-charge induced modulation in the longitudinal phase space of a high-brightness electron bunch. The essence of the effect is in transformation of initial density perturbations into energy modulation along the bunch. Since rf photoinjectors produce a very "cold" electron beam with small intrinsic energy spread, the modulation significantly modifies the energy spectrum.

Using an experimental setup with two accelerator sections and a spectrometer, we recorded the energy spectra of the electron bunch for a wide range of energies. The structure in the measured energy spectra was interpreted as a projection of the modulated longitudinal phase space. This method allowed us to determine the main parameters of modulation (wavelength, energy, and density modulation amplitudes) and their dependences on the beam energy. Numerical simulations based on a longitudinal space-charge model demonstrated reasonable agreement with the measured data. We showed that the longitudinal space-charge force can essentially modify the broadband spectrum of the initial beam density modulation and give rise to a high frequency energy modulation observed by the rf zero-phasing method.

In closing, we note that the observed amplitude of final energy modulation is in the range of $20-40 \mathrm{keV}$, which may exceed the expected intrinsic energy spread $(\sim 8 \mathrm{keV})$ by a large factor. The reconstructed amplitude of the density modulation (a few percent) is comparable with the measured fluctuations of $\mathrm{rf}$ gun drive laser intensity along the $266 \mathrm{~nm}$ laser pulse. Thus, nonuniformities in the longitudinal bunch density in the range of only a few percent can cause a strong distortion in the longitudinal phase space due to the action of the space-charge force. In the DUV-FEL accelerator this distortion happens while a beam with a modest peak current $(\sim 200 \mathrm{~A})$ is traveling along the accelerator of only $15 \mathrm{~m}$. For much longer accelerators with several stages of compression, such as those proposed for X-ray FELs [2,3], this effect can be much stronger and can lead to a significant microbunching instability that degrades the electron beam quality beyond the FEL tolerance. Therefore, the experimental results and their analysis presented in this paper warrant controlled increase of the intrinsic energy spread to damp the microbunching instability for shortwavelength FELs $[12,13]$.

\section{ACKNOWLEDGMENTS}

The authors thank B. Sheehy and H. Loos for their help in carrying out the experiment. We are grateful to D. Dowell, A. Doyuran, P. Emma, W. S. Graves, C. Limborg, 
S. Krinsky, J. B. Murphy, J. Rose, X. J. Wang, J. $\mathrm{Wu}, \mathrm{Z}$. $\mathrm{Wu}$, and L.H.-Yu for useful discussions and comments. The work was performed under DOE Contracts No. DE-AC02-76CH00016 and No. DEAC03-76SF00515.

[1] H. Winick, J. Synchrotron Radiat. 5, 168 (1998).

[2] SLAC Report No. SLAC-R-593, edited by J. Galayda, 2002.

[3] TESLA Report No. TESLA-FEL-2002-09, 2002.

[4] W. Fawley et al., in Proceedings of the 2003 Particle Accelerator Conference, Portland, OR (IEEE, Piscataway, NJ, 2003), p. 923.

[5] W. Graves et al., in Proceedings of the 2003 Particle Accelerator Conference, Portland, OR (Ref. [4]), p. 959.

[6] M. Borland et al., Nucl. Instrum. Methods Phys. Res., Sect. A 483, 268 (2002).

[7] S. Heifets, S. Krinsky, and G. Stupakov, Phys. Rev. ST Accel. Beams 5, 064401 (2002).

[8] Z. Huang and K.-J. Kim, Phys. Rev. ST Accel. Beams 5, 074401 (2002).

[9] E. L. Saldin, E. A. Schneidmiller, and M. V. Yurkov, Nucl. Instrum. Methods Phys. Res., Sect. A 490, 1 (2002).

[10] E. L. Saldin, E. A. Schneidmiller, and M. V. Yurkov, Nucl. Instrum. Methods Phys. Res., Sect. A 483, 516 (2002).

[11] Z. Huang et al., in Proceedings of the 2003 Particle Accelerator Conference, Portland, OR (Ref. [4]). pp. 3138-3140.

[12] E. L. Saldin, E. A. Schneidmiller, and M.V. Yurkov, TESLA Report No. TESLA-FEL-2003-02, 2003; Nucl. Instrum. Methods Phys. Res., Sect. A 528, 355 (2004).
[13] Z. Huang et al., SLAC Report No. SLAC-PUB10334, 2004; Phys. Rev. ST Accel. Beams 7, 074401 (2004).

[14] W. Graves et al., in Proceedings of the 2001 Particle Accelerator Conference, Chicago, IL (IEEE, Piscataway, NJ, 2001), p. 2224.

[15] M. Hüning et al., Nucl. Instrum. Methods Phys. Res., Sect. A 475, 348 (2001).

[16] D. X. Wang et al., Phys. Rev. E 57, 2283 (1998).

[17] H. Loos et al., in Proceedings of the 2002 European Particle Accelerator Conference, Paris, 2002 (EPS-IGA and CERN, Geneva, 2002), p. 814.

[18] T. Shaftan et al., in Proceedings of the 2003 Particle Accelerator Conference, Portland, OR (Ref. [4]), p. 2011.

[19] T. Shaftan et al., Nucl. Instrum. Methods Phys. Res., Sect. A 528, 397 (2004).

[20] Z. Huang and T. Shaftan, Nucl. Instrum. Methods Phys. Res., Sect. A 528, 345 (2004).

[21] T. Shaftan et al., in Proceedings of the 2002 European Particle Accelerator Conference, Paris, 2002 (Ref. [17]), p. 834.

[22] T. Shaftan and L. H. Yu, BNL Report No. BNL-714902003-JA, 2003.

[23] M. Hüening and H. Schlarb, in Proceedings of the 2003 Particle Accelerator Conference, Portland, OR (Ref. [4]), p. 2074.

[24] A. Doyuran (private communication).

[25] C. Limborg (private communication).

[26] R. Bonifacio, C. Pellegrini, and L. M. Narducci, Opt. Commun. 50, 373 (1984).

[27] J. Rosenzweig et al., Report No. DESY-TESLA-FEL96-15. 